\title{
Supragastric Belching: Prevalence and Association With Gastroesophageal Reflux Disease and Esophageal Hypomotility
}

\author{
Nikolaos Koukias, Philip Woodland, Etsuro Yazaki, and Daniel Sifrim* \\ Barts and the London School of Medicine and Dentistry, Queen Mary University of London, UK
}

\begin{abstract}
Background/Aims
Supragastric belching $(\mathrm{SGB})$ is a phenomenon during which air is sucked into the esophagus and then rapidly expelled through the mouth. Patients often complain of severely impaired quality of life. Our objective was to establish the prevalence of excessive SGB within a high-volume gastrointestinal physiology unit, and evaluate its association with symptoms, esophageal motility and gastresophageal reflux disease.
\end{abstract}

\section{Methods}

We established normal values for SGB by analyzing 24-hour pH-impedance in 40 healthy asymptomatic volunteers. We searched 2950 consecutive patient reports from our upper GI Physiology Unit (from 2010-2013) for SGB. Symptoms were recorded by a standardized questionnaire evaluating for reflux, dysphagia, and dyspepsia symptoms. We reviewed the predominant symptoms, 24-hour pH-impedance and high-resolution esophageal manometry results.

\section{Results}

Excessive SGB was defined as $>13$ per 24 hours. We identified 100 patients with excessive SGB. Ninety-five percent of these patients suffered from typical reflux symptoms, $86 \%$ reported excessive belching, and $65 \%$ reported dysphagia. Forty-one percent of patients with excessive SGB had pathological acid reflux. Compared to the patients with normal acid exposure these patients trended towards a higher number of SGB episodes. Forty-four percent of patients had esophageal hypomotility. Patients with hypomotility had a significantly higher frequency of SGB compared to those with normal motility $(118.3 \pm 106.1$ vs $80.6 \pm 75.7, P=0.020$ ).

\section{Conclusions}

Increased belching is rarely a symptom in isolation. Pathological acid exposure and hypomotility are associated with more SGB frequency. Whether SGB is a disordered response to other esophageal symptoms or their cause is unclear.

(J Neurogastroenterol Motil 2015;21:398-403)

Received: January 6, 2015 Revised: March 17, 2015 Accepted: April 15, 2015

(c) This is an Open Access article distributed under the terms of the Creative Commons Attribution Non-Commercial License (http://creativecommons. org/licenses/by-nc/4.0) which permits unrestricted non-commercial use, distribution, and reproduction in any medium, provided the original work is properly cited.

${ }^{*}$ Correspondence: Daniel Sifrim, MD, PhD

Wingate Institute for Neurogastroenterology, 26 Ashfield Street, London E1 2AJ, UK

Tel: +44-0-207-882-2631, Fax: +44-0-207-375-2103, E-mail: d.sifrim@qmul.ac.uk

Financial support: None.

Conflicts of interest: Daniel Sifrim receives a research grant from Reckitt Benckiser UK. Nikolaos Koukias, Philip Woodland, and Etsuro Yazaki have no conflicts of interest to declare.

Author contributions: Nikolaos Koukias was involved in study design, data acquisition and analysis, and drafting of the manuscript; Philip Woodland was involved in the study design, data analysis and interpretation of data, and drafting of the manuscript; Etsuro Yazaki was involved in data acquisition and analysis; and Daniel Sifrim was involved in study design and concept, interpretation of data, drafting of the manuscript, and overall study supervision.

ORCID: Nikolaos Koukias, http://orcid.org/0000-0002-2482-8465; Etsuro Yazaki, http://ircud.org/0000-0002-5315-4957; Daniel Sifrim, http://orcid.org/0000-0002-2482-8465. 
Key Words

Electric impedance; Eructation; Esophageal motility disorders; Gastroesophageal reflux

\section{Introduction}

Excessive belching is a commonly reported symptom. It may occur in the context of gastroesophageal reflux symptoms, with dyspepsia, or can be an isolated symptom. The most common cause of belching is a physiological venting mechanism. Accumulated gas in the proximal stomach stimulates stretch receptors in the gastric wall, initiating a vago-vagal reflex culminating in a transient lower esophageal sphincter relaxation. The consequent "gastric belch" serves to prevent gaseous over-distension of the stomach.

It has long been described that, in some cases, the cause of excessive belching is not due to the gastric belching mechanism, but is instead caused by a rapid process of air entry by "sucking" or "pushing" air from the mouth or pharynx into the esophagus and then expelling it with abdominal straining. ${ }^{1}$ This process can now be easily observed using intra-esophageal impedance, and has been termed supragastric belching (SGB) since the gas does not originate from the stomach, and indeed frequently does not involve gas entry into the stomach. ${ }^{2}$

Patients with SGB often complain of excessive belching with a severe impact on quality of life, and belching can occur hundreds of times per day. SGB is considered to be a behavioral disorder, and in some cases a response to an unpleasant sensation originating from the esophagus or the abdomen. It may be initially performed consciously but at consultation the patient usually has no control over the symptom.

SGB has been studied in selected patients attending gastrointestinal physiology units. For example, a group of patients with gastroesophageal reflux symptoms were found to have a higher number of supragastric belches than healthy controls. ${ }^{3}$ Similarly, high numbers of supragastric belches were seen in patients complaining of excessive belching. ${ }^{2}$

Thus far studies of prevalence and physiological findings in large numbers of patients are lacking.

We aimed to examine the prevalance of pathological SGB in patients attending a high-volume gastrointestinal physiology unit. We also aimed to assess the association of SGB with gastro- esophageal reflux and with esophageal motility disorders as defined by high-resolution manometry (HRM).

\section{Materials and Methods}

\section{Healthy Volunteers}

First, to establish normal values for SGB we analyzed 24-hour $\mathrm{pH}$-impedance studies in 40 healthy asymptomatic volunteers. High-resolution esophageal manonometry (Given Imaging, Yokneam, Israel) was performed initially to identify the position of the lower esophageal sphincter and rule out significant esophageal dysmotility. All healthy volunteers had no upper gastrointestinal symptoms. A combined $\mathrm{pH}$-impedance catheter (Sandhill Scientific, Inc, Highlands Ranch, CO, USA) was placed transnasally such that the antimony esophageal $\mathrm{pH}$ probe was situated $5 \mathrm{~cm}$ above the lower esophageal sphincter. The catheter also has eight electrode rings enabling impedance recording at 6 segments from $3 \mathrm{~cm}$ to $17 \mathrm{~cm}$ above the lower esophageal sphincter. Tracings were manually analysed by one of the authors (N.K.) for presence of reflux and supragastric belches. We defined a SGB according to criteria already described by Bredenoord et $\mathrm{al}^{2}$ (a rapid rise in impedance $(\geq 1000 \Omega$ ) moving in an aboral direction, followed by a return to baseline moving in the opposite direction). SGB less than 5 seconds apart were considered as a single episode. Reflux episodes were considered to be related to SGB if they began within 1 second of a SGB episode. Meal periods were not analysed. Normal reflux values from 24-hour $\mathrm{pH}$-impedance were defined according to previously published accepted criteria. ${ }^{4}$ We subsequently established a SGB frequency above the 95th percentile of normal values as abnormal. $^{5}$

\section{Patients}

We retrospectively searched in our database the reports for the keywords SGB of 2950 consecutive patients attending the Upper GI Physiology Unit of Royal London Hospital between 2010 and 2013. All pH-impedance tracings are manually analyzed at our institution and the presence of SGB is routinely not- 
ed in reports. We manually re-evaluated all tracings identified in the search to confirm those subjects with abnormal levels of SGB (based on the normal values established). Patients had been referred for the assessment of gastroesophageal reflux disease, dysphagia and non-cardiac chest pain. Symptoms were recorded by a standardized questionnaire used in our department. Patients were asked to report whether they had heartburn, regurgitation, dysphagia, retrosternal pain, epigastric pain, excessive belching, excessive cough, hoarsness, and dental erosions, giving details about the frequency and the intensity of their symptoms.

All received high-resolution esophageal manometry and 24hour $\mathrm{pH}$-impedance monitoring using the same equipment as in healthy volunteers after a minimum of 7 days off proton pump inhibitor and prokinetic therapy. We reviewed the predominant symptoms, 24-hour $\mathrm{pH}$-impedance and high resolution esophageal manometry results.

\section{Statistical Methods}

All data is presented as mean $\pm \mathrm{SD}$. Group comparisons are made using the Mann-Whitney test (data is non-parametric). Comparisons of proportions are assessed using Fisher's exact test.

\section{Results}

No healthy volunteers had pathological gastroesophageal reflux on 24-hour $\mathrm{pH}$-impedance. There was a range of 0 to 15 SGB episodes in the 40 healthy volunteers (median 0 , mean 3 ). The 95th percentile value, and hence the upper limit of normal, was calculated at $13 \mathrm{SGB}$ per 24 hours.

We identified 100 patients ( 54 female) with excessive SGB, giving a prevalence of $3.4 \%$ in our referral population. The mean age of these patients was 48 (median 47) and age range was 12 to
84 years. Ninety-five out of 100 patients suffered from typical reflux symptoms (heartburn and regurgitation). Eighty-six patients reported excessive belching with 50 considering that belching was their predominant symptom. Sixty-five patients reported dysphagia, 16 had non-cardiac chest pain, 51 excessive bloating, and 15 epigastric pain. Twenty-nine of the patients had hiatal hernia which was diagnosed with HRM.

The frequency of SGB displayed considerable inter-individual variability. The number of SGB episodes counted within 24 hours ranged between 17 and 510 (median 69, mean 101). The 25 th and the 75 th percentile were 37 and 124 SGB episodes per 24 hours respectively. Forty-one out of 100 patients with excessive SGB had pathological acid exposure ranging from $4.3 \%$ to $54.1 \%$. Compared to the patients with normal acid exposure these patients had a trend towards a higher number of SGB episodes $(109.2 \pm 93.1$ vs $90.1 \pm 93.9, P=0.110), 39$ out of 41 had typical reflux symptoms and 37 reported bothersome belching.

In the patients with increased SGB and pathological acid exposure, we assessed the acid reflux episodes that occurred immediately after (within 1 second) a SGB (Fig. 1). The median number of these episodes was 12.5 (range 0 to 65 ) per patient. Acid exposure time due to reflux episodes occurring immediately after SGB corresponded to a mean $27 \%$ of the total acid exposure time (median $18 \%$, range $0 \%$ to $79 \%$ of the 24 -hour acid exposure) in these patients. ${ }^{5}$

On HRM 44 patients out of 100 had esophageal hypomotility. Thirty-one of them reported dysphagia. According to the Chicago classification ${ }^{6} 18$ had frequent failed peristalsis, 16 had weak peristalsis with large defects, 10 weak peristalsis with small defects. Patients with hypomotility had a significantly higher frequency of SGB compared to the group with normal motility $(118.3 \pm 106.1$ vs $80.6 \pm 75.7, P=0.020$; Fig. 2 and Fig. 3$)$.

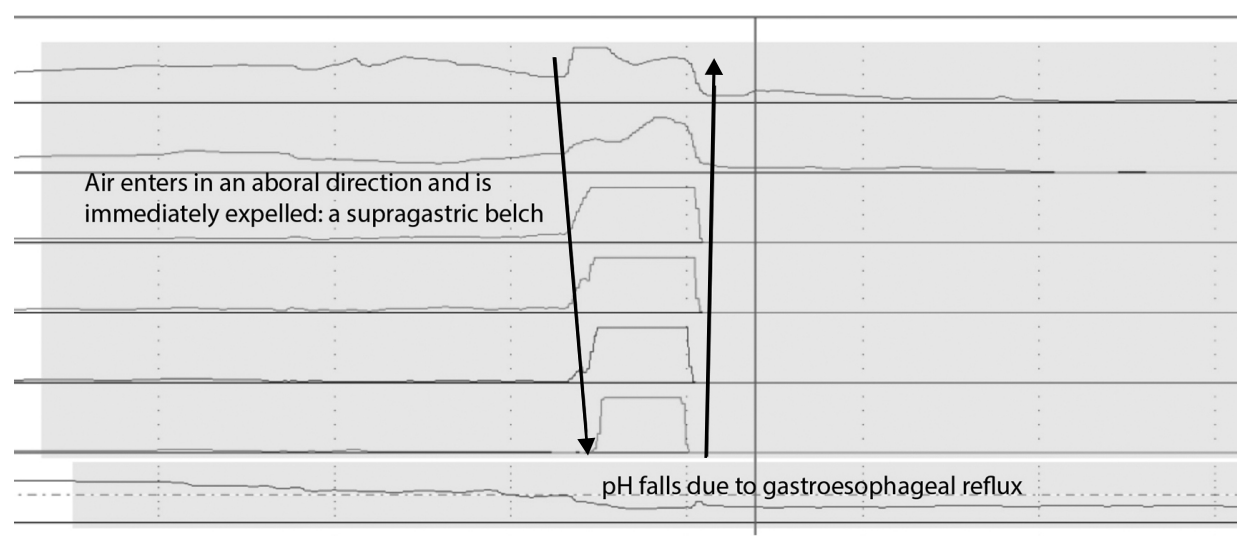

Figure 1. Example $\mathrm{pH}$-impedance tracing showing a supragastric belch followed immediately by a gastresophageal reflux event. 
This was true even when patients with pathological esophageal acid exposure were excluded from analysis $(102.5 \pm 105.6$ vs $68.7 \pm 68.9, P<0.05)$.

In order to distinguish a phenotype of patients that had severe SGB we determined patients with more than 124 SGB episodes per 24 hours ( $>75$ th percentile for the patient group). These 25 patients more often had pathological acid exposure (65\% vs $32 \%, P<0.01)$ and hypomotility (65\% vs $38 \%, P=$ $0.030)$.

\section{Discussion}

This study evaluated SGB in a healthy population, and in a large patient population attending a high-volume gastrointestinal physiology investigation unit. We found that: (1) 13 supragastric

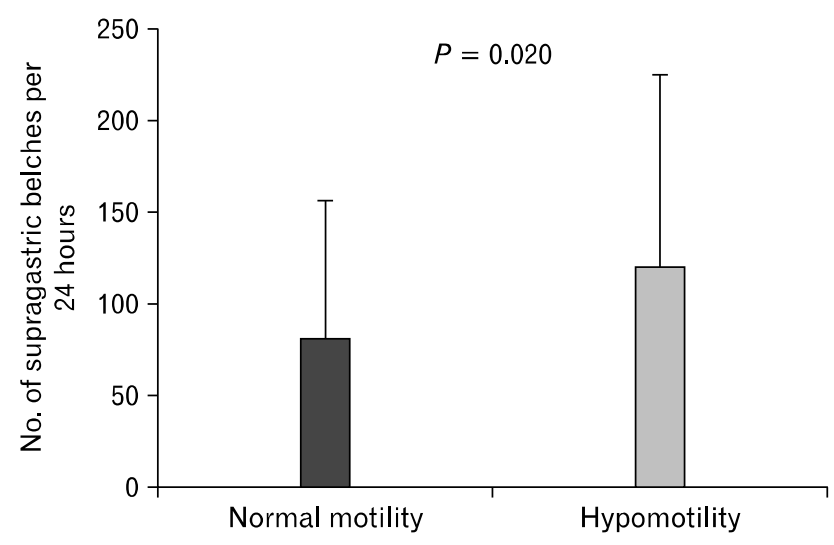

Figure 2. Supragastric belching frequency in patients with esophageal hypomotility and in patients with normal esophageal motility. belches can be considered the upper limit of normal in a healthy population. (2) Excessive SGB was found in $3.4 \%$ of our referral population. (3) Excessive SGB rarely occurs in isolation without other upper gastrointestinal symptoms. (4) Excessive SGB was frequently observed in patients with gastroesophageal reflux disease and in patients with esophageal hypomotility. (5) In some cases, excessive SGB can "contribute” significantly to gastroesophageal reflux.

Over recent years it has been demonstrated that SGB can be accurately assessed using intraluminal impedance techniques. ${ }^{2}$ Previous studies have assessed SGB in defined patients with excessive belching or reflux symptoms, ${ }^{2,3}$ but we believe that this is the first to comprehensively assess symptoms, reflux parameters and esophageal motility in a large- scale, unselected population.

SGB has previously been described in a small $(n=10)$ healthy population, where a median frequency of 2 per 24 hours was discovered. ${ }^{3}$ Our larger healthy population confirm that SGB does occur in healthy subjects, but its frequency is low.

We observed that excessive SGB is relatively infrequent in a patient population, yet in high volume centre such as ours one may expect to encounter it in patients 2 to 3 times per month. It is interesting to note that SGB very rarely existed as an isolated symptom in our population. Only 5 of 100 patients complained only of excessive belching alone. The majority (95\%) complained also of typical reflux symptoms, and $65 \%$ complained of dysphagia. This may support the hypothesis that SGB can be a subconscious and often unhelpful response to an upper gastrointestinal symptom. Surprisingly we found that some patients could have excessive SGB without considering it as a bothersome symptom. Fourteen percent of patients with objective evidence of
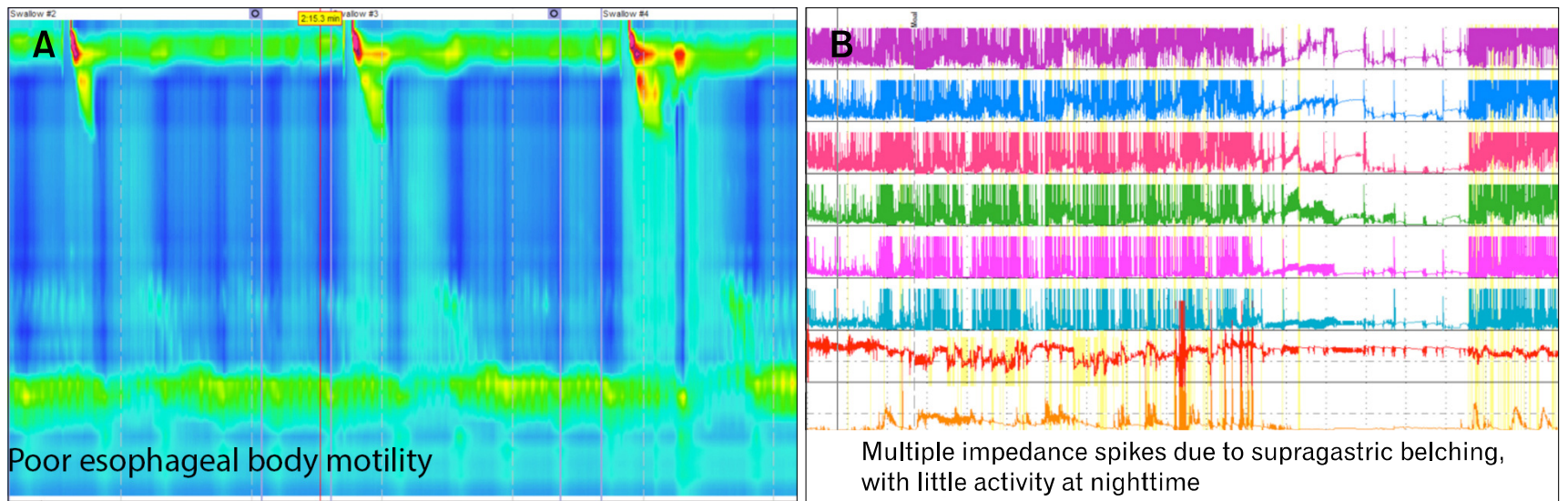

Figure 3. Example high-resolution esophageal manometry plot (A) and 24-hour $\mathrm{pH}$-impedance tracing (B) from a patient with esophageal hypomotility and excessive supragastric belching. 
SGB did not consider themselves to suffer from excessive belching.

This study confirmed the intimate relationship between SGB and gastroesophageal reflux that has been previously described. ${ }^{3}$ Overall, there was only a trend towards increased SGB in patients with reflux disease compared to those without. There is a complex relationship between the two, with reflux being dependent on many factors independent from SGB (eg, hiatus hernia, lower esophageal sphincter pressure, reflux volume, etc). However, in a subgroup of patients, we found that there was a significant contribution to total esophageal acid exposure by reflux episodes that occurred immediately after SGB, thus consolidating the hypothesis that SGB may also play a role in the pathogenesis of gastroesophageal reflux disease. The relationship between SGB and gastroesophageal reflux is complex. On the one hand there is intrathoracic negative pressure induced by an abrupt diaphragmatic aboral movement with initial increase in esophagogastric junction pressure, but this is immediately followed by esophageal distension-induced esophagogastic junction relaxation that allows reflux to occur in some individuals where the barrier is sufficiently overcome. ${ }^{7}$ A study investigating a possible improvement in acid exposure time with behavioral therapy in patients with excessive SGB would be of interest.

We were able to demonstrate that esophageal hypomotility has a relationship with excessive SGB. Within the group of patients with increased SGB, those with esophageal hypomotility on HRM demonstrated a higher number of supragastric belches than patients with normal motility. We wondered whether gastroesophageal reflux could be a confounding factor to explain this, but the association persisted regardless of whether patients had pathological reflux or not. Fifty-eight percent of the patients with excessive acid reflux and 44\% of our total SGB patient group had esophageal hypomotility. This is in contrast to a recent study evaluating esophageal motility in patients with troublesome belching where no such association was seen. However this study was performed with standard (not high resolution) manometry and therefore may have underdiagnosed hypomotility. ${ }^{8}$ Our current study cannot distinguish cause and effect with this relationship. It is possible that hypomotility could lead to symptoms that lead to SGB. Alternatively, it is possible that gaseous distension from SGB could impact on motility patterns.

Future studies could further investigate the association between SGB and hypomotility and evaluate possible improvement after SGB therapy.

Our study identified a group of patients with severe SGB (>
75 th percentile for supragastric belches per 24 hours). This group had more frequent esophageal hypomotility and more frequent gastroesophageal reflux disease. This group of patients may get the greatest benefit from therapy. Patients with SGB have severely impaired health-related quality of life, similar to other functional disorders. ${ }^{2}$ Therefore these patients should be correctly diagnosed and referred for appropriate treatment. Management should include patient's reassurance about the benign cause of their symptoms, since it might relieve their symptom related anxiety and concern. Recession of SGB during sleep, speech and distraction, as well as the type of muscles that are included in its pathogenesis are suggestive of the behavioural nature of the disease. ${ }^{7,9}$ Hence, interest is mainly focused in behavioural therapies. Speech therapy has been applied in a recent pilot study with 11 patients and showed positive results. ${ }^{10}$ Similar positive results have been reported with behavioral therapy in another study. ${ }^{11}$ Baclofen has also been applied with success in a small open label study. ${ }^{12}$

Our study had some limitations. Firstly it is a retrospective study and relies on accurate diagnosis of SGB on the initial reporting. The presence of SGB is actively sought and reported at our unit, so whilst the prevalence may be an underestimate, we expect this effect to be small. Secondly the study group included patients referred to a tertiary referral center, and thus may not be fully representative of a typical gastroenterology patient sample. Nevertheless, we believe that this is the largest cohort of patients with SGB yet to be studied, and as such there is value in our findings.

Increased SGB was identified in 100/2950 patients investigated at the GI physiology Unit over a 4 years period. Increased belching is rarely a symptom in isolation and almost always coexists with other esophageal symptoms, most commonly dysphagia $(65 \%)$ and heartburn/regurgitation (95\%). Pathological acid exposure and hypomotility were associated with more SGB frequency. Whether SGB is a disordered response to other esophageal symptoms or their cause is still unclear. Behavioral therapy and baclofen have shown promising results in patients with predominant belching. The role of therapy for patients with SGB-associated reflux symptoms or dysphagia is under current investigation.

\section{References}

1. Talley NJ, Stanghellini V, Heading RC, Koch KL, Malagelada JR, Tytgat GN. Functional gastroduodenal disorders. Gut 1999; 45(suppl 2):II37-II42. 
2. Bredenoord AJ, Weusten BL, Sifrim D, Timmer R, Smout AJ. Aerophagia, gastric, and supragastric belching: a study using intraluminal electrical impedance monitoring. Gut 2004;53:1561-1565.

3. Hemmink GJ, Bredenoord AJ, Weusten BL, Timmer R, Smout AJ. Supragastric belching in patients with reflux symptoms. Am J Gastroenterol 2009;104:1992-1997.

4. Shay S, Tutuian R, Sifrim D, et al. Twenty-four hour ambulatory simultaneous impedance and $\mathrm{pH}$ monitoring: a multicenter report of normal values from 60 healthy volunteers. Am J Gastroenterol 2004; 99:1037-1043.

5. Koukias N, Jafari J, Yazaki E, Woodland P, Sifrim D. Supragastric belching: prevalence and association with gastroesophageal reflux disease and esophageal hypomotility [abstract]. United European Gastroenterol J 2014;2(suppl 1).

6. Bredenoord AJ, Fox M, Kahrilas PJ, et al. Chicago classification criteria of esophageal motility disorders defined in high resolution esophageal pressure topography. Neurogastroenterol Motil 2012; 24(suppl 1):57-65.

7. Kessing BF, Bredenoord AJ, Smout AJ. Mechanisms of gastric and supragastric belching: a study using concurrent high-resolution manometry and impedance monitoring. Neurogastroenterol Motil 2012;24:e573-e579.

8. Silva AC, Aprile LR, Dantas RO. Esophageal motility in troublesome belching. Arq Gastroenterol 2013;50:107-110.

9. Karamanolis G, Triantafyllou K, Tsiamoulos Z, et al. Effect of sleep on excessive belching: a 24-hour impedance-pH study. J Clin Gastroenterol 2010;44:332-334.

10. Hemmink GJ, Ten Cate L, Bredenoord AJ, Timmer R, Weusten $\mathrm{BL}$, Smout AJ. Speech therapy in patients with excessive supragastric belching--a pilot study. Neurogastroenterol Motil 2010;22:24-28, e2-e3.

11. Katzka DA. Simple office-based behavioral approach to patients with chronic belching. Dis Esophagus 2013;26:570-573.

12. Blondeau K, Boecxstaens V, Rommel N, et al. Baclofen improves symptoms and reduces postprandial flow events in patients with rumination and supragastric belching. Clin Gastroenterol Hepatol 2012;10:379-384. 